\section{The biological and clinical challenge of liver cancer heterogeneity}

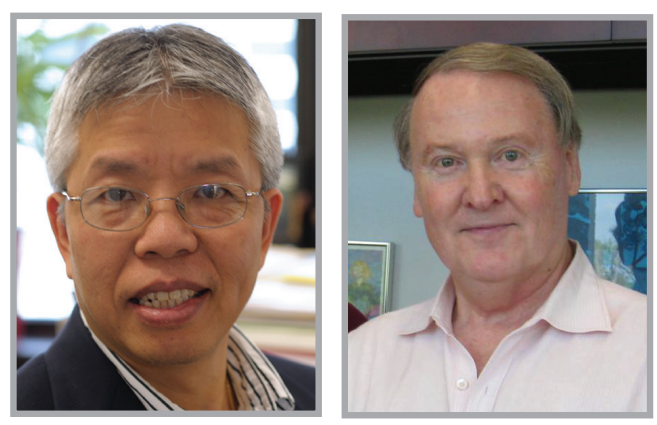

Xin Wei Wang ${ }^{*, 1} \&$ Snorri S Thorgeirsson ${ }^{2}$

Primary liver cancer (PLC) consists of two major histological subtypes: hepatocellular carcinoma (HCC) and intrahepatic cholangiocarcinoma (ICC). PLC is, similar to all major human cancers, clinically and biologically heterogeneous, and highly resistant to treatment. Consequently, 5 -year survival rates of PLC in the US has been $<20 \%$ over the past 40 years with little sign of improvement [1]. Patient ethnicity, underlying liver diseases due to various etiological factors such as viral hepatitis resulting from hepatitis $\mathrm{B}$ and hepatitis $\mathrm{C}$ infection, parasitic infection, alcoholic liver disease, or obesity, etc., may contribute to molecular and biological heterogeneity [2]. Furthermore, recent application of genomic technologies (e.g., transcriptomic and sequencing analyses) have reinforced the extent of heterogeneity in PLC [2]. In addition, the heterogeneous hepatic microenvironment in PLC resulting from inflammation and varying degrees of liver fibrosis and cirrhosis may also affect tumor biology. The major barrier to improve outcome of patients with PLC is the incomplete understanding of PLC heterogeneity and the inability to subdivide the patients into biologically and genomically unique subgroups harboring well-defined and effective-treatment targets, a central strategy applied by the precision cancer medicine (PCM) approach. PCM utilizes cancer genomic data with precision based on mechanistic understanding of a particular state of tumor biology to facilitate rational treatment choices that are tailored to individual patients. It is paramount to reevaluate current practice guidelines and to apply the PCM strategy for managing PLC patients and designing new clinical trials.

\section{Factors influencing tumor \\ heterogeneity}

The extensive heterogeneity of PLC can be attributed to the presence of multiple and complex etiological factors, extensive genomic complexity and underling tumor biology (Figure 1). Age, gender and ethnicity are known risk factors for HCC [3]. Environmental factors such as chronic infection by hepatitis B, hepatitis C and parasites as well as chemical carcinogens such as aflatoxin B1 are also recognized as major etiological factors for the

\section{KEYWORDS}

- cholangiocarcinoma

- hepatocellular carcinoma

- molecular subtype - precision cancer medicine • tumor genomics heterogeneity

\section{"Precision cancer medicine utilizes cancer genomic data with precision based on mechanistic understanding of a particular state of tumor biology to facilitate rational treatment choices that are tailored to individual patients."}

'Laboratory of Human Carcinogenesis, Center for Cancer Research, National Cancer Institute, Bethesda, MD 20892, USA 2Laboratory of Experimental Carcinogenesis, Center for Cancer Research, National Cancer Institute, Bethesda, MD 20892, USA

*Author for correspondence: xw3u@nih.gov
Future $\mathrm{fSS}$ 


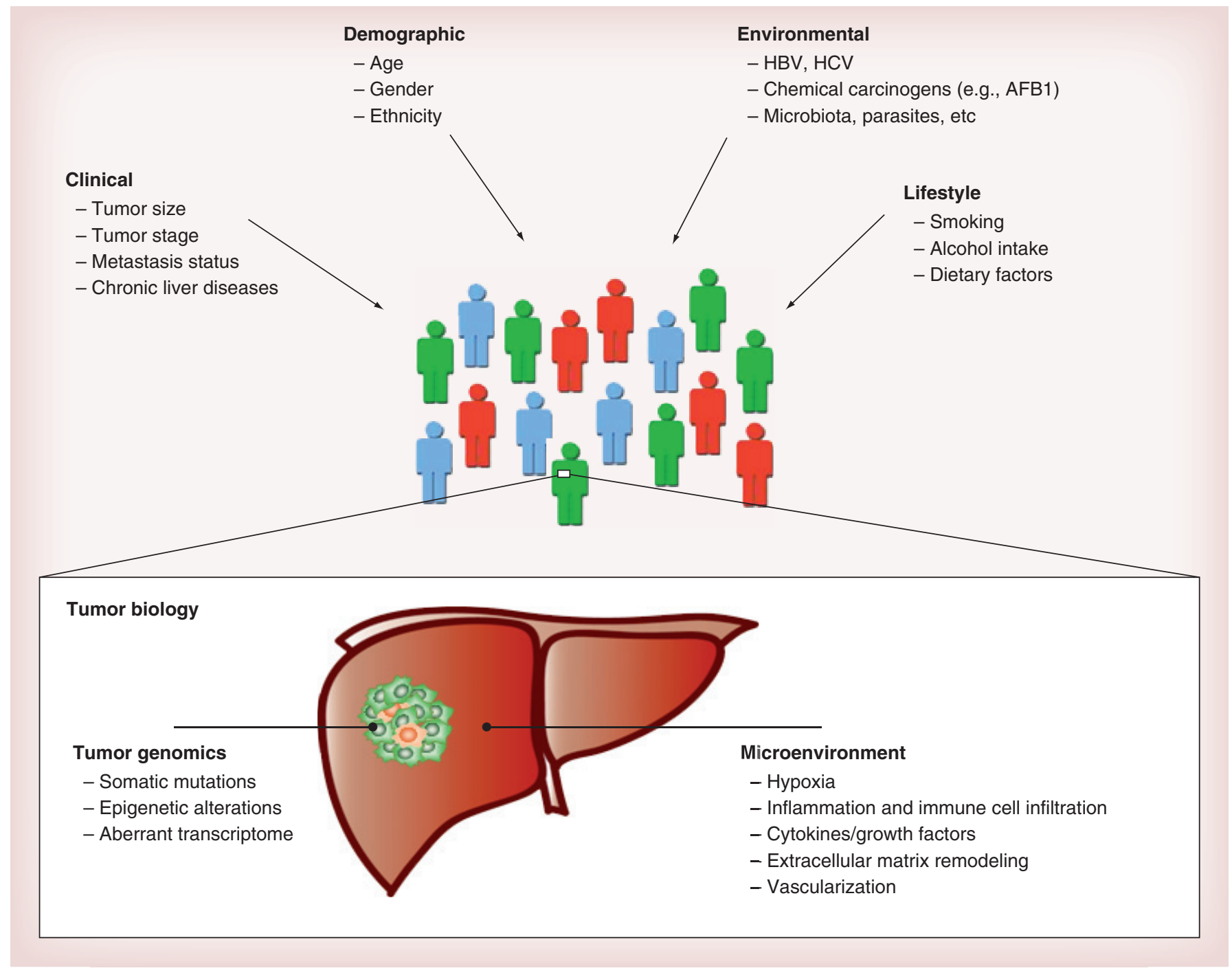

Figure 1. The etiology and features of liver cancer heterogeneity.

development of PLC [3]. In addition, unhealthy lifestyle such as cigarette smoking, excess alcohol intake and dietary factors are closely associated with PLC. Different etiological factors can, in principle, drive the development of PLC through different molecular mechanisms, resulting in differences in clinical presentation and underling tumor biology [4]. In addition to tumor genomics, patient's outcomes can be exacerbated by the presence of chronic liver diseases due to various etiological factors [5]. Such a compounding effect makes PLC one of the most difficult-totreat malignancies, and constitutes an enormous challenge to clinical hepatic oncology.

Because of the vast inter-tumor heterogeneity in human tumors, one of the major goals is to identify specific druggable cancer driver genes whose functions are essential for the fitness of cancer cells within a defined tumor subgroup. It is known that genome-wide investigations may be effective in defining tumor subgroups since all cancers arise as a result of somatically acquired changes in the DNA of cancer cells [6]. Consequently, next generation sequencing technologies have been applied to HCC and ICC studies with the goal to identify tumor subgroups and key cancer drivers [7]. These studies are exciting and encouraging to define functional pathways that pave the way for new molecular classification for clinical application. However, one major issue this approach faces is that because of tumor evolution, each solid tumor carries thousands of somatic genome alterations accumulated over time as documented in the 
COSMIC database and elsewhere, and a majority of mutations are noncontributing passengers whose functions are not essential to tumor cells at the time a tumor specimen is procured and analyzed $[8,9]$. The presence of considerable somatic changes found in solid tumors may explain the increased genomic intra-tumor heterogeneity and poses additional challenges to accurately define druggable targets in individual subtypes [10].

\section{Precision cancer medicine}

The application of the PCM design in which diagnosis, patient classification and treatment response are based on molecular classification obtained by an integrated systems biology strategy can significantly contribute to successful outcome in hepatic oncology (Figure 2). A concerted effort to develop a well-defined biobank and information commons, and an implementation of biomarker-guided interventions is essential. To link functions of cellular genes to PLC, numerous global gene-expression profiling analyses over the last decade have been carried out to profile PLC with different ethnicity and etiologies [11,12]. These studies are successful in defining stable PLC prognostic subgroups and identifying key functional pathways. To improve molecular precision, an integrated omics strategy through the integration of multiple levels of omics data has been explored with some successes $[2,13]$. Integrative genomics, through the combination of exonic mutations and somatic copy number alteration (SCNA) data, have shown promise in revealing candidate drivers linked to colorectal and lung cancer [14,15]. Integration of somatic copy-number alteration and transcriptome as well as metabolites allows successful identification of HCC subgroups and functional drivers [16-19]. This approach also led to the development of a companion diagnostic test that may be useful to select HCC patients who may benefit from post-resection adjuvant IFN- $\alpha$ treatment [20,21]. It is reasonable to expect that an integrated systems biology strategy will be effective in addressing tumor heterogeneity and improve outcomes for liver cancer patients.

\section{Tumor biopsy wanted}

Tumor biopsy is an invaluable resource that allows clinicians to make accurate diagnoses and sensible choices for cancer treatment. For example, tumor biospecimens are routinely collected in clinical studies involving most common cancers such as those in lung, breast, prostate and colon. Tumor biopsy has been paramount for the success in the identification of a subset of non-small-cell lung cancer patients with EGFR mutations who have a favorable response to gefitinib [22] and a subset of melanoma patients with BRAF mutations who have a favorable response to vemurafenib [23]. A successful development of breast cancer subtype algorithm is also based in the availability of tumor specimens routinely collected for diagnoses [24]. While traditional histological examination of tumor needle biopsy offers definitive diagnosis of HCC, a potential risk for seeding of tumor cells along the needle tract has led to the development of noninvasive diagnosis criteria. Imaging techniques offer a noninvasive mean and have been important in patient management during last decade since they are powerful in potentially detecting early HCC that may be eligible for potentially curative treatments, such as orthotopic liver transplantation, surgical resection, percutaneous ethanol injection, or radiofrequency ablation [25]. The discovery of unique radiological features of HCC detected by helical-computed tomography and gadolinium magnetic resonance imaging has led to the recommendation by EASL as noninvasive diagnosis criteria incorporating into practice guidelines for the management of HCC [26]. As such, most of the clinical studies to evaluate HCC treatment responses are not required to collect tumor biospecimens. It sounds paradoxical: on one hand the recommended practice guideline offers a great noninvasive tool to accurately diagnose HCC, but on the other hand eliminates our ability to assess tumor biology and identify biomarkers for both guiding and improving therapies. The ability to examine tumor biopsy could help identifying a subset of HCC patients with a favorable response to a particular treatment. For example, an initial assessment of the results from a Phase II study to evaluate tivantinib as second-line treatment of advanced HCC showed that the trial did not meet its main objective. However, subsequent analyses revealed that a subset of c-Met positive HCC had a significant improvement of overall survival in the treatment arm [27]. Thus, weighing the risks and benefits, it is conceivable that collecting biospecimens in clinical studies adds a significant value by providing 'real-time' assessment with precision and mechanistic clarity. It seems that the current practice guidelines constitute a roadblock for the advancement of HCC
"It is reasonable to expect that an integrated systems biology strategy will be effective in addressing tumor heterogeneity and improve outcomes for liver cancer patients." 


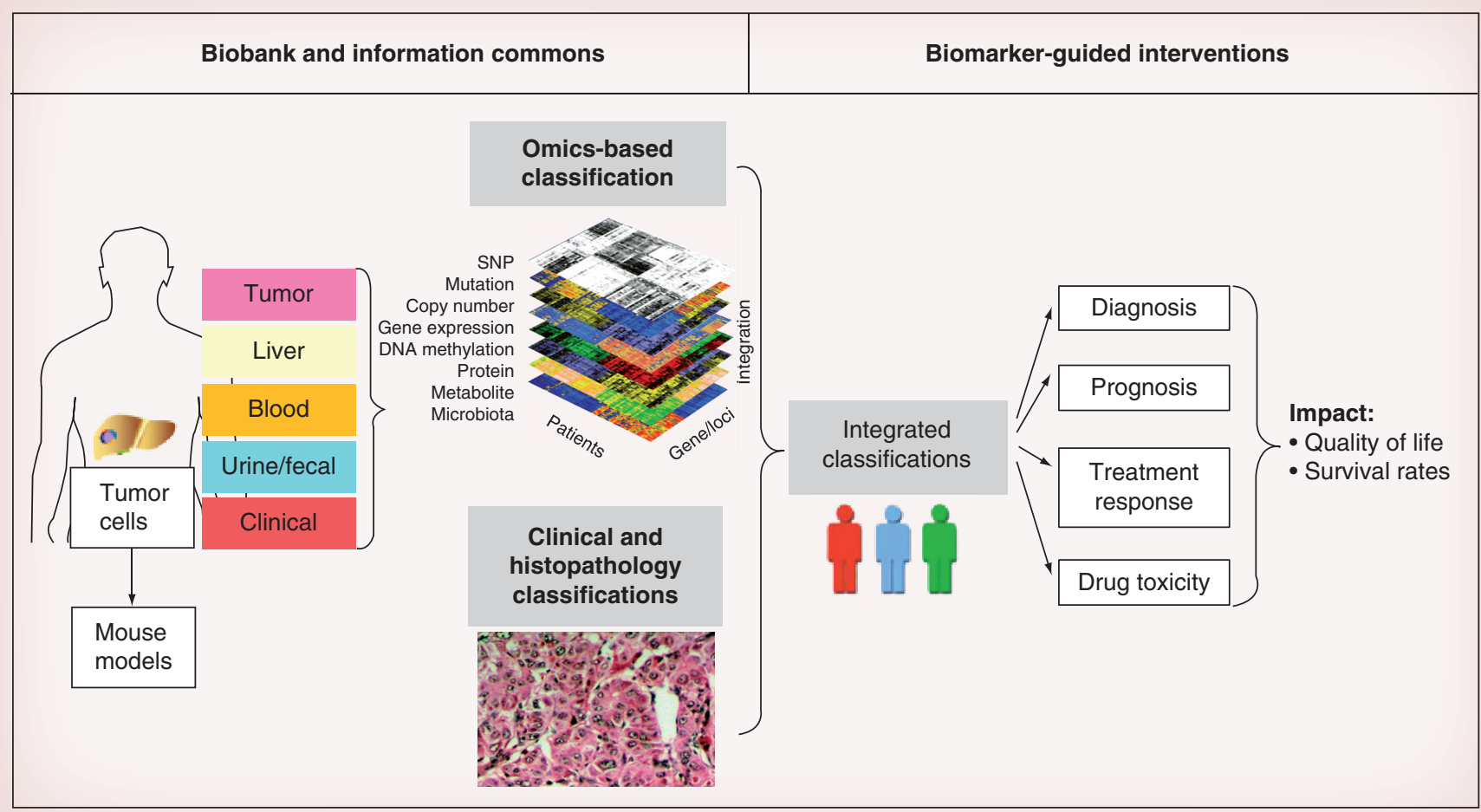

Figure 2. An integrated systems biology strategy to address tumor heterogeneity and improve outcome for liver cancer patients. SNP: Single nucleotide polymorphism.

management. Given the importance of accurate classification through analyzing tumor biology, it is sensible to incorporate the tumor biopsy requirement into current clinical intervention studies in PLC.

\section{Conclusion \& future perspective}

Advanced PLC typically does not respond to available systemic treatment modalities such as chemo and radiotherapies. The success of sorafenib treatment of HCC in the initial SHARP trial in 2008 [28] has revolutionized and energized hepatic oncology practice. Sorafenib, a broad tyrosine kinase inhibitor, offers a statistically significant improvement of overall survival by extending an average of 2.8 months for advanced HCC patients. Since then, more than 80 molecularly targeted agents have been evaluated in Phase II and Phase III trials [29]. However, while these agents are designed to target specific signaling pathways, trial designs generally adopt a one-size-fit-all strategy without biomarker-guided patient selection. This may explain recent failures in multiple Phase III studies for HCC since the successful SHARP trial. Such setbacks reflect the disappointing state of the current PLC management and emphasize the need to incorporate PCM strategy into clinical practice.

A key advantage provided by PCM is the development of robust biologically and clinically relevant tumor biomarkers which functionally can act as cancer drivers for molecular targeting and tools for precise classification of patients. A roadmap for clinical translation of diagnostic biomarkers has been suggested to include four steps: identification, validation, assay development and clinical implementation [12]. It is clear that in order to evaluate tumor biomarkers, each step requires the use of highquality tumor biospecimens preferably derived from randomized control studies to minimize false positivity. The hepatic oncology practice suffers significantly from our inability to identify and validate candidate tumor biomarkers in readily available and accessible tumor biopsies from randomized control studies, a practice commonly associated with lung and breast cancer practice. The development of biobank and information commons is essential for the implementation of PCM-based management of PLC patients. This is consistent with the report 
commissioned by the National Academy of Sciences at the request of the Director of the $\mathrm{NIH}$ [30]. The appalling global health impact of PLC requires international collaborations for the development of well-defined liver cancer consortia that can contribute to improve understanding of both liver carcinogenesis and consensus clinical management for this dreadful disease.
Financial \& competing interests disclosure

The authors have no relevant affliations or financial involvement with any organization or entity with a financial interest in or financial conflict with the subject matter or materials discussed in the manuscript. This includes employment, consultancies, honoraria, stock ownership or options, expert testimony, grants or patents received or pending, or royalties.

No writing assistance was utilized in the production of this manuscript.

\section{References}

1 Siegel R, Naishadham D, Jemal A. Cancer statistics, 2013. CA Cancer J. Clin. 63, 11-30 (2013).

2 Roessler S, Budhu A, Wang XW. Deciphering cancer heterogeneity: the biological space. Front. Cell Dev. Biol. 2(12), $1-5$ (2014).

3 El-Serag HB, Rudolph KL. Hepatocellular carcinoma: epidemiology and molecular carcinogenesis. Gastroenterology 132, 2557-2576 (2007).

4 Thorgeirsson SS, Grisham JW. Molecular pathogenesis of human hepatocellular carcinoma. Nat. Genet. 31, 339-346 (2002).

5 Budhu A, Wang X W. The role of cytokines in hepatocellular carcinoma. J. Leukoc. Biol. 80, 1197-1213 (2006).

6 Stratton MR, Campbell PJ, Futreal PA. The cancer genome. Nature 458, 719-724 (2009).

7 Shibata T, Aburatani H. Exploration of liver cancer genomes. Nat. Rev. Gastroenterol. Hepatol. 11(6), 340-349 (2014).

8 Beroukhim R, Mermel CH, Porter D et al. The landscape of somatic copy-number alteration across human cancers. Nature 463 , 899-905 (2010).

9 Forbes SA, Bindal N, Bamford S et al. COSMIC: mining complete cancer genomes in the Catalogue of Somatic Mutations in Cancer. Nucleic Acids Res. 39, D945-D950 (2011).

10 Gerlinger M, Rowan AJ, Horswell S et al. Intratumor heterogeneity and branched evolution revealed by multiregion sequencing. N. Engl. J. Med. 366, 883-892 (2012).

11 Breuhahn K, Gores G, Schirmacher P. Strategies for hepatocellular carcinoma therapy and diagnostics: lessons learned from high throughput and profiling approaches. Hepatology 53, 2112-2121 (2011).
12 Hoshida Y, Moeini A, Alsinet C et al. Gene signatures in the management of hepatocellular carcinoma. Semin. Oncol. 39, 473-485 (2012).

13 Lawrence MS, Stojanov P, Polak P et al. Mutational heterogeneity in cancer and the search for new cancer-associated genes. Nature 499, 214-218 (2013).

14 Cancer Genome Atlas Research Network. Comprehensive molecular characterization of human colon and rectal cancer. Nature 487, 330-337 (2012).

15 Cancer Genome Atlas Research Network. Comprehensive genomic characterization of squamous cell lung cancers. Nature 489, 519-525 (2012)

16 Woo HG, Park ES, Lee JS et al. Identification of potential driver genes in human liver carcinoma by genomewide screening. Cancer Res. 69, 4059-4066 (2009).

17 Roessler S, Long EL, Budhu A et al. Integrative genomic identification of genes on $8 \mathrm{p}$ associated with hepatocellular carcinoma progression and patient survival. Gastroenterology 142, 957-966 (2012).

18 Budhu A, Roessler S, Zhao X et al. Integrated metabolite and gene expression profiles identify lipid biomarkers associated with progression of hepatocellular carcinoma and patient outcomes. Gastroenterology 144, 1066-1075 (2013).

19 Ozdemir B, Abd-Almageed W, Roessler S et al. iSubgraph: integrative genomics for subgroup discovery in hepatocellular carcinoma using graph mining and mixture models. PLoS ONE 8, e78624 (2013).

20 Ji J, Yu L, Yu Z et al. Development of a miR-26 companion diagnostic test for adjuvant interferon-alpha therapy in hepatocellular carcinoma. Int. J. Biol. Sci. 9, 303-312 (2013).

21 Ji J, Shi J, Budhu A et al. MicroRNA expression, survival, and response to interferon in liver cancer. N. Engl. J. Med. 361, 1437-1447 (2009).

22 Paez JG, Janne PA, Lee JC et al. EGFR mutations in lung cancer: correlation with clinical response to gefitinib therapy. Science 304, 1497-1500 (2004).

23 Chapman PB, Hauschild A, Robert C et al. Improved survival with vemurafenib in melanoma with BRAF V600E mutation. N. Engl. J. Med. 364, 2507-2516 (2011).

24 Cancer Genome Atlas Research Network. Comprehensive molecular portraits of human breast tumours. Nature 490, 61-70 (2012).

25 Tan CH, Low SC, Thng CH. APASL and AASLD consensus guidelines on imaging diagnosis of hepatocellular carcinoma: a review. Int. J. Hepatol. 2011, 519783 (2011).

26 Bruix J, Sherman M, Llovet JM et al. Clinical management of hepatocellular carcinoma. Conclusions of the Barcelona-2000 EASL conference. European Association for the Study of the Liver. J. Hepatol. 35, 421-430 (2001).

27 Santoro A, Rimassa L, Borbath I et al. Tivantinib for second-line treatment of advanced hepatocellular carcinoma: a randomized, placebo-controlled Phase 2 study. Lancet Oncol. 14, 55-63 (2013).

28 Llovet JM, Ricci S, Mazzaferro V et al. Sorafenib in advanced hepatocellular carcinoma. N. Engl. J. Med. 359, 378-390 (2008).

29 ClinicalTrials.gov. www.clinicaltrials.gov

30 National Research Council (US) Committee on A Framework forDeveloping a New Taxonomy of Disease. Toward Precision Medicine: Building a Knowledge Network for Biomedical Research and a New Taxonomy of Disease. The National Academies Press, DC, USA (2011). 